\title{
Asymptotic behavior of solutions of mixed type impulsive neutral differential equations
}

\author{
Jessada Tariboon ${ }^{1 *}$, Sotiris K Ntouyas ${ }^{2,3}$ and Chatthai Thaiprayoon ${ }^{1}$
}

"Correspondence:
jessadat@kmutnb.ac.th
${ }^{1}$ Nonlinear Dynamic Analysis
Research Center, Department of
Mathematics, Faculty of Applied
Science, King Mongkut's University
of Technology North Bangkok,
Bangkok, 10800, Thailand
Full list of author information is
available at the end of the article

available at the end of the article

\begin{abstract}
This paper investigates the asymptotic behavior of solutions of the mixed type neutral differential equation with impulsive perturbations $[x(t)+C(t) x(t-\tau)-D(t) x(\alpha t)]^{\prime}+P(t) f(x(t-\delta))+\frac{Q(t)}{t} x(\beta t)=0,0<t_{0} \leq t, t \neq t_{k}$, $x\left(t_{k}\right)=b_{k} x\left(t_{k}^{-}\right)+\left(1-b_{k}\right)\left(\int_{t_{k}-\delta}^{t_{k}} P(s+\delta) f(x(s)) d s+\int_{\beta t_{k}}^{t_{k}} \frac{Q(s / \beta)}{s} x(s) d s\right), k=1,2,3, \ldots$ Sufficient conditions are obtained to guarantee that every solution tends to a constant as $t \rightarrow \infty$. Examples illustrating the abstract results are also presented.

MSC: 34K25; 34K45

Keywords: asymptotic behavior; nonlinear neutral delay differential equation; impulse; Lyapunov functional
\end{abstract}

\section{Introduction}

The main purpose of this paper is to investigate the asymptotic behavior of solutions of the following mixed type neutral differential equation with impulsive perturbations:

$$
\left\{\begin{array}{l}
{[x(t)+C(t) x(t-\tau)-D(t) x(\alpha t)]^{\prime}+P(t) f(x(t-\delta))+\frac{Q(t)}{t} x(\beta t)=0,} \\
\quad 0<t_{0} \leq t, t \neq t_{k}, \\
x\left(t_{k}\right)=b_{k} x\left(t_{k}^{-}\right)+\left(1-b_{k}\right)\left(\int_{t_{k}-\delta}^{t_{k}} P(s+\delta) f(x(s)) d s+\int_{\beta t_{k}}^{t_{k}} \frac{Q(s / \beta)}{s} x(s) d s\right), \\
\quad k=1,2,3, \ldots,
\end{array}\right.
$$

where $\tau, \delta>0,0<\alpha, \beta<1, C(t), D(t) \in P C\left(\left[t_{0}, \infty\right), \mathbb{R}\right), P(t), Q(t) \in P C\left(\left[t_{0}, \infty\right), \mathbb{R}_{0}^{+}\right), f \in$ $C(\mathbb{R}, \mathbb{R}), 0<t_{k}<t_{k+1}$ with $\lim _{k \rightarrow \infty} t_{k}=\infty$ and $b_{k}, k=1,2,3, \ldots$, are given constants. For $J \subset \mathbb{R}, P C(J, \mathbb{R})$ denotes the set of all functions $h: J \rightarrow \mathbb{R}$ such that $h$ is continuous for $t_{k} \leq t<t_{k+1}$ and $\lim _{t \rightarrow t_{k}^{-}} h(t)=h\left(t_{k}^{-}\right)$exists for all $k=1,2, \ldots$.

The theory of impulsive differential equations appears as a natural description of several real processes subject to certain perturbations whose duration is negligible in comparison with the duration of the process. Differential equations involving impulse effects occurs in many applications: physics, population dynamics, ecology, biological systems, biotechnology, industrial robotic, pharmacokinetics, optimal control, etc. The reader may refer, for instance, to the monographs by Bainov and Simeonov [1], Lakshmikantham et al. [2], Samoilenko and Perestyuk [3], and Benchohra et al. [4]. In recent years, there has been increasing interest in the oscillation, asymptotic behavior, and stability theory of impulsive delay differential equations and many results have been obtained (see [5-20] and the references cited therein).

(2014 Tariboon et al.; licensee Springer. This is an Open Access article distributed under the terms of the Creative Commons Attribution License (http://creativecommons.org/licenses/by/2.0), which permits unrestricted use, distribution, and reproduction in any medium, provided the original work is properly cited. 
Let us mention some papers from which are motivation for our work. By the construction of Lyapunov functionals, the authors in [8] studied the asymptotic behavior of solutions of the nonlinear neutral delay differential equation under impulsive perturbations,

$$
\left\{\begin{array}{l}
{[x(t)+C(t) x(t-\tau)]^{\prime}+P(t) f(x(t-\delta))=0, \quad 0<t_{0} \leq t, t \neq t_{k},} \\
x\left(t_{k}\right)=b_{k} x\left(t_{k}^{-}\right)+\left(1-b_{k}\right) \int_{t_{k}-\delta}^{t_{k}} P(s+\delta) f(x(s)) d s, \quad k=1,2,3, \ldots
\end{array}\right.
$$

A similar method was used in [21] by considering an impulsive Euler type neutral delay differential equation with similar impulsive perturbations

$$
\left\{\begin{array}{l}
{[x(t)-D(t) x(\alpha t)]^{\prime}+\frac{Q(t)}{t} x(\beta t)=0, \quad 0<t_{0} \leq t, t \neq t_{k},} \\
x\left(t_{k}\right)=b_{k} x\left(t_{k}^{-}\right)+\left(1-b_{k}\right) \int_{\beta t_{k}}^{t_{k}} \frac{Q(s / \beta)}{s} x(s) d s, \quad k=1,2,3, \ldots
\end{array}\right.
$$

In this paper we combine the two papers $[8,21]$ and we study the mixed type impulsive neutral differential equation problem (1.1). By using a similar method with the help of four Lyapunov functionals, sufficient conditions are obtained to guarantee that every solution of (1.1) tends to a constant as $t \rightarrow \infty$. We note that problems (1.2) and (1.3) can be derived from the problem (1.1) as special cases, i.e., if $D(t) \equiv 0$ and $Q(t) \equiv 0$, then (1.1) reduces to (1.2) while if $C(t) \equiv 0$ and $P(t) \equiv 0$, then (1.1) reduces to (1.3). Therefore, the mixed type of nonlinear delay with an Euler form of impulsive neutral differential equations gives more general results than the previous one.

Setting $\eta_{1}=\max \{\tau, \delta\}, \eta_{2}=\min \{\alpha, \beta\}$, and $\eta=\min \left\{t_{0}-\eta_{1}, \eta_{2} t_{0}\right\}$, we define an initial function as

$$
x(t)=\varphi(t), \quad t \in\left[\eta, t_{0}\right],
$$

where $\varphi \in P C\left(\left[\eta, t_{0}\right], \mathbb{R}\right)=\left\{\varphi:\left[\eta, t_{0}\right] \rightarrow \mathbb{R} \mid \varphi\right.$ is continuous everywhere except at a finite number of point $s$, and $\varphi\left(s^{-}\right)$and $\varphi\left(s^{+}\right)=\lim _{s \rightarrow s^{+}} \varphi(s)$ exist with $\left.\varphi\left(s^{+}\right)=\varphi(s)\right\}$.

A function $x(t)$ is said to be a solution of (1.1) satisfying the initial condition (1.4) if

(i) $x(t)=\varphi(t)$ for $\eta \leq t \leq t_{0}, x(t)$ is continuous for $t \geq t_{0}$ and $t \neq t_{k}, k=1,2,3, \ldots$;

(ii) $x(t)+C(t) x(t-\tau)-D(t) x(\alpha t)$ is continuously differentiable for $t>t_{0}, t \neq t_{k}$, $k=1,2,3, \ldots$, and satisfies the first equation of system (1.1);

(iii) $x\left(t_{k}^{+}\right)$and $x\left(t_{k}^{-}\right)$exist with $x\left(t_{k}^{+}\right)=x\left(t_{k}\right)$ and satisfy the second equation of system (1.1).

A solution of (1.1) is said to be nonoscillatory if it is eventually positive or eventually negative. Otherwise, it is said to be oscillatory.

\section{Main results}

We are now in a position to establish our main results.

Theorem 2.1 Assume that:

$\left(\mathrm{H}_{1}\right)$ There exists a constant $M>0$ such that

$$
|x| \leq|f(x)| \leq M|x|, \quad x \in \mathbb{R}, x f(x)>0, \text { for } x \neq 0 .
$$


$\left(\mathrm{H}_{2}\right)$ The functions $C$, D satisfy

$$
\lim _{t \rightarrow \infty}|C(t)|=\mu<1, \quad \lim _{t \rightarrow \infty}|D(t)|=\gamma<1 \quad \text { with } \mu+\gamma<1,
$$

and

$$
C\left(t_{k}\right)=b_{k} C\left(t_{k}^{-}\right), \quad D\left(t_{k}\right)=b_{k} D\left(t_{k}^{-}\right) .
$$

$\left(\mathrm{H}_{3}\right) t_{k}-\tau$ and $\alpha t_{k}$ are not impulsive points, $0<b_{k} \leq 1, k=1,2, \ldots$, and $\sum_{k=1}^{\infty}\left(1-b_{k}\right)<\infty$.

$\left(\mathrm{H}_{4}\right)$ The functions $P$, Q satisfy

$$
\left\{\begin{array}{c}
\lim \sup _{t \rightarrow \infty}\left[\int_{t-\delta}^{t+\delta} P(s+\delta) d s+\int_{\beta t}^{t+\delta} \frac{Q(s / \beta)}{s} d s\right. \\
\left.+\mu\left(1+\frac{P(t+\tau+\delta)}{P(t+\delta)}\right)+\gamma\left(1+\frac{P((t / \alpha)+\delta)}{\alpha P(t+\delta)}\right)\right]<\frac{2}{M}
\end{array}\right.
$$

and

$$
\left\{\begin{array}{r}
\lim \sup _{t \rightarrow \infty}\left[\int_{t-\delta}^{t / \beta} P(s+\delta) d s+\int_{\beta t}^{t / \beta} \frac{Q(s / \beta)}{s} d s\right. \\
\left.+\mu\left(1+\frac{t Q((t+\tau) / \beta)}{(t+\tau) Q(t / \beta)}\right)+\gamma\left(1+\frac{Q(t /(\alpha \beta))}{Q(t / \beta)}\right)\right]<2 .
\end{array}\right.
$$

Then every solution of (1.1) tends to a constant as $t \rightarrow \infty$.

Proof Let $x(t)$ be any solution of system (1.1). We will prove that the $\lim _{t \rightarrow \infty} x(t)$ exists and is finite. Indeed, the system (1.1) can be written as

$$
\begin{aligned}
& {\left[x(t)+C(t) x(t-\tau)-D(t) x(\alpha t)-\int_{t-\delta}^{t} P(s+\delta) f(x(s)) d s-\int_{\beta t}^{t} \frac{Q(s / \beta)}{s} x(s) d s\right]^{\prime}} \\
& +P(t+\delta) f(x(t))+\frac{Q(t / \beta)}{t} x(t)=0, \quad t \geq t_{0}, t \neq t_{k}, \\
& x\left(t_{k}\right)=b_{k} x\left(t_{k}^{-}\right)+\left(1-b_{k}\right)\left(\int_{t_{k}-\delta}^{t_{k}} P(s+\delta) f(x(s)) d s\right. \\
& \left.\quad+\int_{\beta t_{k}}^{t_{k}} \frac{Q(s / \beta)}{s} x(s) d s\right), \quad k=1,2, \ldots .
\end{aligned}
$$

From $\left(\mathrm{H}_{2}\right)$ and $\left(\mathrm{H}_{4}\right)$, we choose constants $\varepsilon, \lambda, v, \rho>0$ sufficiently small such that $\mu+\varepsilon<1$ and $\gamma+\lambda<1$ and $T>t_{0}$ sufficiently large, for $t \geq T$,

$$
\begin{aligned}
& {\left[\int_{t-\delta}^{t+\delta} P(s+\delta) d s+\int_{\beta t}^{t+\delta} \frac{Q(s / \beta)}{s} d s+(\mu+\varepsilon)\left(1+\frac{P(t+\tau+\delta)}{P(t+\delta)}\right)\right.} \\
& \left.\quad+(\gamma+\lambda)\left(1+\frac{P((t / \alpha)+\delta)}{\alpha P(t+\delta)}\right)\right] \leq \frac{2}{M}-v, \\
& {\left[\int_{t-\delta}^{t / \beta} P(s+\delta) d s+\int_{\beta t}^{t / \beta} \frac{Q(s / \beta)}{s} d s+(\mu+\varepsilon)\left(1+\frac{t Q((t+\tau) / \beta)}{(t+\tau) Q(t / \beta)}\right)\right.} \\
& \left.\quad+(\gamma+\lambda)\left(1+\frac{Q(t /(\alpha \beta))}{Q(t / \beta)}\right)\right] \leq 2-\rho,
\end{aligned}
$$

and, for $t \geq T$,

$$
|C(t)| \leq \mu+\varepsilon, \quad|D(t)| \leq \gamma+\lambda .
$$


From (2.1), (2.10), we have

$$
\frac{|C(t)|}{\mu+\varepsilon} \leq 1 \leq \frac{f^{2}(x(t-\tau))}{x^{2}(t-\tau)}, \quad \frac{|D(t)|}{\gamma+\lambda} \leq 1 \leq \frac{f^{2}(x(\alpha t))}{x^{2}(\alpha t)}, \quad t \geq T
$$

which lead to

$$
\begin{aligned}
& |C(t)| x^{2}(t-\tau) \leq(\mu+\varepsilon) f^{2}(x(t-\tau)), \\
& |D(t)| x^{2}(\alpha t) \leq(\gamma+\lambda) f^{2}(x(\alpha t)), \quad t \geq T .
\end{aligned}
$$

In the following, for convenience, the expressions of functional equalities and inequalities will be written without its domain. This means that the relations hold for all sufficiently large $t$.

Let $V(t)=V_{1}(t)+V_{2}(t)+V_{3}(t)+V_{4}(t)$, where

$$
\begin{aligned}
V_{1}(t)= & {\left[x(t)+C(t) x(t-\tau)-D(t) x(\alpha t)-\int_{t-\delta}^{t} P(s+\delta) f(x(s)) d s-\int_{\beta t}^{t} \frac{Q(s / \beta)}{s} x(s) d s\right]^{2}, } \\
V_{2}(t)= & \int_{t-\delta}^{t} P(s+2 \delta) \int_{s}^{t} P(u+\delta) f^{2}(x(u)) d u d s \\
& +\int_{\beta t}^{t} \frac{P((s+\beta \delta) / \beta)}{\beta} \int_{s}^{t} \frac{Q(u / \beta)}{u} x^{2}(u) d u d s, \\
V_{3}(t)= & \int_{t-\delta}^{t} \frac{Q((s+\delta) / \beta)}{s+\delta} \int_{s}^{t} P(u+\delta) f^{2}(x(u)) d u d s \\
& +\int_{\beta t}^{t} \frac{Q\left(s / \beta^{2}\right)}{s} \int_{s}^{t} \frac{Q(u / \beta)}{u} x^{2}(u) d u d s,
\end{aligned}
$$

and

$$
\begin{aligned}
V_{4}(t)= & (\mu+\varepsilon) \int_{t-\tau}^{t} P(s+\tau+\delta) f^{2}(x(s)) d s+(\mu+\varepsilon) \int_{t-\tau}^{t} \frac{Q((s+\tau) / \beta)}{s+\tau} x^{2}(s) d s \\
& +(\gamma+\lambda) \int_{\alpha t}^{t} \frac{Q(s /(\alpha \beta))}{s} x^{2}(s) d s+\frac{\gamma+\lambda}{\alpha} \int_{\alpha t}^{t} P((s / \alpha)+\delta) f^{2}(x(s)) d s .
\end{aligned}
$$

Computing $d V_{1} / d t$ along the solution of (1.1) and using the inequality $2 a b \leq a^{2}+b^{2}$, we have

$$
\begin{aligned}
\frac{d V_{1}}{d t}= & -2[x(t)+C(t) x(t-\tau)-D(t) x(\alpha t) \\
& \left.-\int_{t-\delta}^{t} P(s+\delta) f(x(s)) d s-\int_{\beta t}^{t} \frac{Q(s / \beta)}{s} x(s) d s\right] \\
& \times\left(P(t+\delta) f(x(t))+\frac{Q(t / \beta)}{t} x(t)\right) \\
\leq & -P(t+\delta)\left[2 x(t) f(x(t))-|C(t)| x^{2}(t-\tau)-|C(t)| f^{2}(x(t))-|D(t)| x^{2}(\alpha t)\right. \\
& -|D(t)| f^{2}(x(t))-\int_{t-\delta}^{t} P(s+\delta) f^{2}(x(s)) d s-f^{2}(x(t)) \int_{t-\delta}^{t} P(s+\delta) d s
\end{aligned}
$$




$$
\begin{aligned}
& \left.-\int_{\beta t}^{t} \frac{Q(s / \beta)}{s} x^{2}(s) d s-f^{2}(x(t)) \int_{\beta t}^{t} \frac{Q(s / \beta)}{s} d s\right] \\
& -\frac{Q(t / \beta)}{t}\left[2 x^{2}(t)-|C(t)| x^{2}(t)-|C(t)| x^{2}(t-\tau)-|D(t)| x^{2}(t)\right. \\
& -|D(t)| x^{2}(\alpha t)-\int_{t-\delta}^{t} P(s+\delta) f^{2}(x(s)) d s-x^{2}(t) \int_{t-\delta}^{t} P(s+\delta) d s \\
& \left.-\int_{\beta t}^{t} \frac{Q(s / \beta)}{s} x^{2}(s) d s-x^{2}(t) \int_{\beta t}^{t} \frac{Q(s / \beta)}{s} d s\right] .
\end{aligned}
$$

Calculating directly for $d V_{i} / d t, i=2,3,4, t \neq t_{k}$, we have

$$
\begin{aligned}
\frac{d V_{2}}{d t}= & P(t+\delta) f^{2}(x(t)) \int_{t-\delta}^{t} P(s+2 \delta) d s-P(t+\delta) \int_{t-\delta}^{t} P(s+\delta) f^{2}(x(s)) d s \\
& +\frac{Q(t / \beta)}{\beta t} x^{2}(t) \int_{\beta t}^{t} P((s+\beta \delta) / \beta) d s-P(t+\delta) \int_{\beta t}^{t} \frac{Q(s / \beta)}{s} x^{2}(s) d s, \\
\frac{d V_{3}}{d t}= & P(t+\delta) f^{2}(x(t)) \int_{t-\delta}^{t} \frac{Q((s+\delta) / \beta)}{s+\delta} d s-\frac{Q(t / \beta)}{t} \int_{t-\delta}^{t} P(s+\delta) f^{2}(x(s)) d s \\
& +\frac{Q(t / \beta)}{t} x^{2}(t) \int_{\beta t}^{t} \frac{Q\left(s / \beta^{2}\right)}{s} d s-\frac{Q(t / \beta)}{t} \int_{\beta t}^{t} \frac{Q(s / \beta)}{s} x^{2}(s) d s,
\end{aligned}
$$

and

$$
\begin{aligned}
\frac{d V_{4}}{d t}= & (\mu+\varepsilon) P(t+\tau+\delta) f^{2}(x(t))-(\mu+\varepsilon) P(t+\delta) f^{2}(x(t-\tau)) \\
& +\frac{(\mu+\varepsilon)}{(t+\tau)} Q((t+\tau) / \beta) x^{2}(t)-\frac{(\mu+\varepsilon)}{t} Q(t / \beta) x^{2}(t-\tau) \\
& +(\gamma+\lambda) \frac{Q(t /(\alpha \beta))}{t} x^{2}(t)-(\gamma+\lambda) \frac{Q(t / \beta)}{t} x^{2}(\alpha t) \\
& +\frac{(\gamma+\lambda)}{\alpha} P((t / \alpha)+\delta) f^{2}(x(t))-(\gamma+\lambda) P(t+\delta) f^{2}(x(\alpha t)) .
\end{aligned}
$$

Summing for $d V_{i} / d t, i=1,2,3$, we obtain

$$
\begin{aligned}
\frac{d V_{1}}{d t}+ & \frac{d V_{2}}{d t}+\frac{d V_{3}}{d t} \\
\leq & -P(t+\delta)\left[2 x(t) f(x(t))-|C(t)| x^{2}(t-\tau)-|C(t)| f^{2}(x(t))-|D(t)| x^{2}(\alpha t)\right. \\
& -|D(t)| f^{2}(x(t))-f^{2}(x(t)) \int_{t-\delta}^{t} P(s+\delta) d s-f^{2}(x(t)) \int_{\beta t}^{t} \frac{Q(s / \beta)}{s} d s \\
& \left.-f^{2}(x(t)) \int_{t-\delta}^{t} P(s+2 \delta) d s-f^{2}(x(t)) \int_{t-\delta}^{t} \frac{Q((s+\delta) / \beta)}{s+\delta} d s\right] \\
& -\frac{Q(t / \beta)}{t}\left[2 x^{2}(t)-|C(t)| x^{2}(t)-|C(t)| x^{2}(t-\tau)-|D(t)| x^{2}(t)\right. \\
& -|D(t)| x^{2}(\alpha t)-x^{2}(t) \int_{t-\delta}^{t} P(s+\delta) d s-x^{2}(t) \int_{\beta t}^{t} \frac{Q(s / \beta)}{s} d s \\
& \left.-\frac{x^{2}(t)}{\beta} \int_{\beta t}^{t} P((s+\beta \delta) / \beta) d s-x^{2}(t) \int_{\beta t}^{t} \frac{Q\left(s / \beta^{2}\right)}{s} d s\right] .
\end{aligned}
$$


Since

$$
\begin{aligned}
& \int_{t-\delta}^{t} P(s+2 \delta) d s=\int_{t}^{t+\delta} P(s+\delta) d s, \quad \int_{\beta t}^{t} \frac{Q\left(s / \beta^{2}\right)}{s} d s=\int_{t}^{t / \beta} \frac{Q(s / \beta)}{s} d s, \\
& \int_{t-\delta}^{t} \frac{Q((s+\delta) / \beta)}{s+\delta} d s=\int_{t}^{t+\delta} \frac{Q(s / \beta)}{s} d s, \quad \frac{1}{\beta} \int_{\beta t}^{t} P((s+\beta \delta) / \beta) d s=\int_{t}^{t / \beta} P(s+\delta) d s,
\end{aligned}
$$

it follows that

$$
\begin{aligned}
\frac{d V_{1}}{d t}+ & \frac{d V_{2}}{d t}+\frac{d V_{3}}{d t} \\
\leq & -P(t+\delta)\left[2 x(t) f(x(t))-|C(t)| x^{2}(t-\tau)-|C(t)| f^{2}(x(t))-|D(t)| x^{2}(\alpha t)\right. \\
& \left.-|D(t)| f^{2}(x(t))-f^{2}(x(t)) \int_{t-\delta}^{t+\delta} P(s+\delta) d s-f^{2}(x(t)) \int_{\beta t}^{t+\delta} \frac{Q(s / \beta)}{s} d s\right] \\
& -\frac{Q(t / \beta)}{t}\left[2 x^{2}(t)-|C(t)| x^{2}(t)-|C(t)| x^{2}(t-\tau)-|D(t)| x^{2}(t)\right. \\
& \left.-|D(t)| x^{2}(\alpha t)-x^{2}(t) \int_{t-\delta}^{t / \beta} P(s+\delta) d s-x^{2}(t) \int_{\beta t}^{t / \beta} \frac{Q(s / \beta)}{s} d s\right] .
\end{aligned}
$$

Adding the above inequality with $d V_{4} / d t$ and using condition (2.11), we have

$$
\begin{aligned}
& \frac{d V_{1}}{d t}+\frac{d V_{2}}{d t}+\frac{d V_{3}}{d t}+\frac{d V_{4}}{d t} \\
& \leq-P(t+\delta)\left[2 x(t) f(x(t))-|C(t)| f^{2}(x(t))-|D(t)| f^{2}(x(t))\right. \\
& \left.-f^{2}(x(t)) \int_{t-\delta}^{t+\delta} P(s+\delta) d s-f^{2}(x(t)) \int_{\beta t}^{t+\delta} \frac{Q(s / \beta)}{s} d s\right] \\
& -\frac{Q(t / \beta)}{t}\left[2 x^{2}(t)-|C(t)| x^{2}(t)-|D(t)| x^{2}(t)\right. \\
& \left.-x^{2}(t) \int_{t-\delta}^{t / \beta} P(s+\delta) d s-x^{2}(t) \int_{\beta t}^{t / \beta} \frac{Q(s / \beta)}{s} d s\right] \\
& +(\mu+\varepsilon) P(t+\tau+\delta) f^{2}(x(t))+\frac{(\mu+\varepsilon)}{t+\tau} Q((t+\tau) / \beta) x^{2}(t) \\
& +(\gamma+\lambda) \frac{Q(t /(\alpha \beta))}{t} x^{2}(t)+\frac{(\gamma+\lambda)}{\alpha} P((t / \alpha)+\delta) f^{2}(x(t)) .
\end{aligned}
$$

Applying (2.8), (2.9), and (2.10), it follows that

$$
\begin{aligned}
\frac{d V}{d t}= & \frac{d V_{1}}{d t}+\frac{d V_{2}}{d t}+\frac{d V_{3}}{d t}+\frac{d V_{4}}{d t} \\
\leq & -P(t+\delta) f^{2}(x(t))\left[\frac{2 x(t)}{f(x(t))}-|C(t)|-|D(t)|-\int_{t-\delta}^{t+\delta} P(s+\delta) d s\right. \\
& \left.-\int_{\beta t}^{t+\delta} \frac{Q(s / \beta)}{s} d s-(\mu+\varepsilon) \frac{P(t+\tau+\delta)}{P(t+\delta)}-\frac{(\gamma+\lambda)}{\alpha} \frac{P((t / \alpha)+\delta)}{P(t+\delta)}\right] \\
& -\frac{Q(t / \beta)}{t} x^{2}(t)\left[2-|C(t)|-|D(t)|-\int_{t-\delta}^{t / \beta} P(s+\delta) d s\right.
\end{aligned}
$$




$$
\begin{aligned}
& \left.-\int_{\beta t}^{t / \beta} \frac{Q(s / \beta)}{s} d s-\frac{(\mu+\varepsilon) t}{t+\tau} \frac{Q((t+\tau) / \beta)}{Q(t / \beta)}-(\gamma+\lambda) \frac{Q(t /(\alpha \beta))}{Q(t / \beta)}\right] \\
\leq & -P(t+\delta) f^{2}(x(t))\left[\frac{2}{M}-\int_{t-\delta}^{t+\delta} P(s+\delta) d s-\int_{\beta t}^{t+\delta} \frac{Q(s / \beta)}{s} d s\right. \\
& \left.-(\mu+\varepsilon)\left(1+\frac{P(t+\tau+\delta)}{P(t+\delta)}\right)-(\gamma+\lambda)\left(1+\frac{P((t / \alpha)+\delta)}{\alpha P(t+\delta)}\right)\right] \\
& -\frac{Q(t / \beta)}{t} x^{2}(t)\left[2-\int_{t-\delta}^{t / \beta} P(s+\delta) d s-\int_{\beta t}^{t / \beta} \frac{Q(s / \beta)}{s} d s\right. \\
& \left.-(\mu+\varepsilon)\left(1+\frac{t}{t+\tau} \frac{Q((t+\tau) / \beta)}{Q(t / \beta)}\right)-(\gamma+\lambda)\left(1+\frac{Q(t /(\alpha \beta))}{Q(t / \beta)}\right)\right] \\
\leq & -P(t+\delta) f^{2}(x(t)) v-\frac{Q(t / \beta)}{t} x^{2}(t) \rho .
\end{aligned}
$$

For $t=t_{k}$, we have

$$
\begin{aligned}
V_{1}\left(t_{k}\right)= & {\left[x\left(t_{k}\right)+C\left(t_{k}\right) x\left(t_{k}-\tau\right)-D\left(t_{k}\right) x\left(\alpha t_{k}\right)\right.} \\
& \left.-\int_{t_{k}-\delta}^{t_{k}} P(s+\delta) f(x(s)) d s-\int_{\beta t_{k}}^{t_{k}} \frac{Q(s / \beta)}{s} x(s) d s\right]^{2} \\
= & {\left[b_{k} x\left(t_{k}^{-}\right)+b_{k} C\left(t_{k}^{-}\right) x\left(t_{k}^{-}-\tau\right)-b_{k} D\left(t_{k}^{-}\right) x\left(\alpha t_{k}^{-}\right)\right.} \\
& \left.-b_{k}\left(\int_{t_{k}-\delta}^{t_{k}} P(s+\delta) f(x(s)) d s+\int_{\beta t_{k}}^{t_{k}} \frac{Q(s / \beta)}{s} x(s) d s\right)\right]^{2} \\
= & b_{k}^{2} V_{1}\left(t_{k}^{-}\right) .
\end{aligned}
$$

It is easy to see that $V_{2}\left(t_{k}\right)=V_{2}\left(t_{k}^{-}\right), V_{3}\left(t_{k}\right)=V_{3}\left(t_{k}^{-}\right)$, and $V_{4}\left(t_{k}\right)=V_{4}\left(t_{k}^{-}\right)$.

Therefore,

$$
\begin{aligned}
V\left(t_{k}\right) & =V_{1}\left(t_{k}\right)+V_{2}\left(t_{k}\right)+V_{3}\left(t_{k}\right)+V_{4}\left(t_{k}\right) \\
& =b_{k}^{2} V_{1}\left(t_{k}^{-}\right)+V_{2}\left(t_{k}^{-}\right)+V_{3}\left(t_{k}^{-}\right)+V_{4}\left(t_{k}^{-}\right) \\
& \leq V_{1}\left(t_{k}^{-}\right)+V_{2}\left(t_{k}^{-}\right)+V_{3}\left(t_{k}^{-}\right)+V_{4}\left(t_{k}^{-}\right) \\
& =V\left(t_{k}^{-}\right) .
\end{aligned}
$$

From (2.12) and (2.13), we conclude that $V(t)$ is decreasing. In view of the fact that $V(t) \geq 0$, we have $\lim _{t \rightarrow \infty} V(t)=\psi$ exist and $\psi \geq 0$.

By using (2.8), (2.9), (2.12), and (2.13), we have

$$
v \int_{T}^{\infty} P(t+\delta) f^{2}(x(t)) d t+\rho \int_{T}^{\infty} \frac{Q(t / \beta)}{t} x^{2}(t) d t \leq V(T)
$$

which yields

$$
P(t+\delta) f^{2}(x(t)), \frac{Q(t / \beta)}{t} x^{2}(t) \in L^{1}\left(t_{0}, \infty\right) .
$$


Hence, for any $\phi>0$ and $\xi \in(0,1)$, we get

$$
\lim _{t \rightarrow \infty} \int_{t-\phi}^{t} P(s+\delta) f^{2}(x(s)) d s=0, \quad \lim _{t \rightarrow \infty} \int_{\xi t}^{t} \frac{Q(s / \beta)}{s} x^{2}(s) d s=0 .
$$

Thus, it follows from (2.4) and (2.5) that

$$
\begin{aligned}
& \int_{t-\delta}^{t} P(s+2 \delta) \int_{s}^{t} P(u+\delta) f^{2}(x(u)) d u d s \\
& \quad+\int_{\beta t}^{t} \frac{P((s+\beta \delta) / \beta)}{\beta} \int_{s}^{t} \frac{Q(u / \beta)}{u} x^{2}(u) d u d s \\
& \leq \int_{t-\delta}^{t+\delta} P(s+\delta) d s \int_{t-\delta}^{t} P(u+\delta) f^{2}(x(u)) d u \\
& \quad+\int_{t-\delta}^{t / \beta} P(s+\delta) d s \int_{\beta t}^{t} \frac{Q(u / \beta)}{u} x^{2}(u) d u \\
& \leq \frac{2}{M} \int_{t-\delta}^{t} P(u+\delta) f^{2}(x(u)) d u d s \\
&+\frac{2}{M} \int_{\beta t}^{t} \frac{Q(u / \beta)}{u} x^{2}(u) d u \rightarrow 0, \quad \text { as } t \rightarrow \infty \\
& \int_{t-\delta}^{t} \frac{Q((s+\delta) / \beta)}{s+\delta} \int_{s}^{t} P(u+\delta) f^{2}(x(u)) d u d s \\
&+\int_{\beta t}^{t} \frac{2\left(s / \beta^{2}\right)}{s} \int_{s}^{t} \frac{Q(u / \beta)}{u} x^{2}(u) d u d s \\
& \leq \int_{\beta t}^{t+\delta} \frac{Q(s / \beta)}{s} d s \int_{t-\delta}^{t} P(u+\delta) f^{2}(x(u)) d u \\
&+\int_{\beta t}^{t / \beta} \frac{Q(s / \beta)}{s} d s \int_{\beta t}^{t} \frac{Q(u / \beta)}{u} x^{2}(u) d u \\
& \\
&+2(u / \beta) \\
& \\
&
\end{aligned}
$$

and

$$
\begin{aligned}
(\mu+\varepsilon) & \int_{t-\tau}^{t} P(s+\tau+\delta) f^{2}(x(s)) d s+(\mu+\varepsilon) \int_{t-\tau}^{t} \frac{Q((s+\tau) / \beta)}{s+\tau} x^{2}(s) d s \\
& +(\gamma+\lambda) \int_{\alpha t}^{t} \frac{Q(s /(\alpha \beta))}{s} x^{2}(s) d s+\frac{\gamma+\lambda}{\alpha} \int_{\alpha t}^{t} P((s / \alpha)+\delta) f^{2}(x(s)) d s \\
= & (\mu+\varepsilon) \int_{t-\tau}^{t} \frac{P(s+\tau+\delta)}{P(s+\delta)} P(s+\delta) f^{2}(x(s)) d s \\
& +(\mu+\varepsilon) \int_{t-\tau}^{t} \frac{s Q((s+\tau) / \beta)}{Q(s / \beta)(s+\tau)} \cdot \frac{Q(s / \beta)}{s} x^{2}(s) d s \\
& +(\gamma+\lambda) \int_{\alpha t}^{t} \frac{Q(s /(\alpha \beta))}{Q(s / \beta)} \cdot \frac{Q(s / \beta)}{s} x^{2}(s) d s
\end{aligned}
$$




$$
\begin{aligned}
& +\frac{\gamma+\lambda}{\alpha} \int_{\alpha t}^{t} \frac{P((s / \alpha)+\delta)}{P(s+\delta)} P(s+\delta) f^{2}(x(s)) d s \\
\leq & \frac{2}{M} \int_{t-\tau}^{t} P(s+\delta) f^{2}(x(s)) d s+2 \int_{t-\tau}^{t} \frac{Q(s / \beta)}{s} x^{2}(s) d s \\
& +2 \int_{\alpha t}^{t} \frac{Q(s / \beta)}{s} x^{2}(s) d s+\frac{2}{M} \int_{\alpha t}^{t} P(s+\delta) f^{2}(x(s)) d s \rightarrow 0, \quad \text { as } t \rightarrow \infty .
\end{aligned}
$$

Therefore, from the above estimations, we have $\lim _{t \rightarrow \infty} V_{2}(t)=0, \lim _{t \rightarrow \infty} V_{3}(t)=0$, and $\lim _{t \rightarrow \infty} V_{4}(t)=0$, respectively.

Thus, $\lim _{t \rightarrow \infty} V_{1}(t)=\lim _{t \rightarrow \infty} V(t)=\psi$, that is,

$$
\begin{aligned}
\lim _{t \rightarrow \infty}[x(t)+C(t) x(t-\tau)-D(t) x(\alpha t) \\
\left.\quad-\int_{t-\delta}^{t} P(s+\delta) f(x(s)) d s-\int_{\beta t}^{t} \frac{Q(s / \beta)}{s} x(s) d s\right]^{2}=\psi .
\end{aligned}
$$

Now, we will prove that the limit

$$
\begin{aligned}
\lim _{t \rightarrow \infty}[x(t)+C(t) x(t-\tau)-D(t) x(\alpha t) \\
\left.\quad-\int_{t-\delta}^{t} P(s+\delta) f(x(s)) d s-\int_{\beta t}^{t} \frac{Q(s / \beta)}{s} x(s) d s\right]
\end{aligned}
$$

exists and is finite. Setting

$$
\begin{aligned}
y(t)= & x(t)+C(t) x(t-\tau)-D(t) x(\alpha t) \\
& -\int_{t-\delta}^{t} P(s+\delta) f(x(s)) d s-\int_{\beta t}^{t} \frac{Q(s / \beta)}{s} x(s) d s,
\end{aligned}
$$

and using (1.1) and condition $\left(\mathrm{H}_{3}\right)$, we have

$$
\begin{aligned}
y\left(t_{k}\right)= & x\left(t_{k}\right)+C\left(t_{k}\right) x\left(t_{k}-\tau\right)-D\left(t_{k}\right) x\left(\alpha t_{k}\right) \\
& -\int_{t_{k}-\delta}^{t_{k}} P(s+\delta) f(x(s)) d s-\int_{\beta t_{k}}^{t_{k}} \frac{Q(s / \beta)}{s} x(s) d s \\
= & b_{k}\left[x\left(t_{k}^{-}\right)+C\left(t_{k}^{-}\right) x\left(t_{k}^{-}-\tau\right)-D\left(t_{k}^{-}\right) x\left(\alpha t_{k}^{-}\right)\right. \\
& \left.-\int_{t_{k}-\delta}^{t_{k}} P(s+\delta) f(x(s)) d s-\int_{\beta t_{k}}^{t_{k}} \frac{Q(s / \beta)}{s} x(s) d s\right] \\
= & b_{k} y\left(t_{k}^{-}\right) .
\end{aligned}
$$

In view of (2.14), it follows that

$$
\lim _{t \rightarrow \infty} y^{2}(t)=\psi
$$

In addition, from (2.16) and (2.17), system (2.6)-(2.7) can be written as

$$
\left\{\begin{array}{l}
y^{\prime}(t)+P(t+\delta) f(x(t))+\frac{Q(t / \beta)}{t} x(t)=0, \quad 0<t_{0} \leq t, t \neq t_{k}, \\
y\left(t_{k}\right)=b_{k} y\left(t_{k}^{-}\right), \quad k=1,2,3, \ldots
\end{array}\right.
$$


If $\psi=0$, then $\lim _{t \rightarrow \infty} y(t)=0$. If $\psi>0$, then there exists a sufficiently large $T^{*}$ such that $y(t) \neq 0$ for any $t>T^{*}$. Otherwise, there is a sequence $\left\{a_{k}\right\}$ with $\lim _{k \rightarrow \infty} a_{k}=\infty$ such that $y\left(a_{k}\right)=0$, and so $y^{2}\left(a_{k}\right) \rightarrow 0$ as $k \rightarrow \infty$. This contradicts $\psi>0$. Therefore, for any $t_{k}>T^{*}$, and $t \in\left[t_{k}, t_{k+1}\right)$, we have $y(t)>0$ or $y(t)<0$ from the continuity of $y$ on $\left[t_{k}, t_{k+1}\right)$. Without loss of generality, we assume that $y(t)>0$ on $\left[t_{k}, t_{k+1}\right)$. It follows from $\left(\mathrm{H}_{3}\right)$ that $y\left(t_{k+1}\right)=b_{k} y\left(t_{k+1}^{-}\right)>0$, and thus $y(t)>0$ on $\left[t_{k+1}, t_{k+2}\right)$. By using mathematical induction, we deduce that $y(t)>0$ on $\left[t_{k}, \infty\right)$. Therefore, from (2.14), we have

$$
\begin{aligned}
\lim _{t \rightarrow \infty} y(t)= & \lim _{t \rightarrow \infty}[x(t)+C(t) x(t-\tau)-D(t) x(\alpha t) \\
& \left.-\int_{t-\delta}^{t} P(s+\delta) f(x(s)) d s-\int_{\beta t}^{t} \frac{Q(s / \beta)}{s} x(s) d s\right]=\kappa,
\end{aligned}
$$

where $\kappa=\sqrt{\psi}$ and is finite. In view of (2.18), for sufficient large $t$, we have

$$
\begin{array}{r}
\int_{\beta t-\delta}^{t} P(s+\delta) f(x(s)) d s+\int_{\beta t-\delta}^{t} \frac{Q(s / \beta)}{s} x(s) d s \\
=y(\beta t-\delta)-y(t)-\sum_{\beta t-\delta<t_{k}<t}\left[y\left(t_{k}\right)-y\left(t_{k}^{-}\right)\right] \\
=y(\beta t-\delta)-y(t)-\sum_{\beta t-\delta<t_{k}<t}\left(1-b_{k}\right) y\left(t_{k}^{-}\right) .
\end{array}
$$

Taking $t \rightarrow \infty$ and using $\left(\mathrm{H}_{3}\right)$, we have

$$
\lim _{t \rightarrow \infty}\left[\int_{\beta t-\delta}^{t} P(s+\delta) f(x(s)) d s+\int_{\beta t-\delta}^{t} \frac{Q(s / \beta)}{s} x(s) d s\right]=0,
$$

which leads to

$$
\lim _{t \rightarrow \infty} \int_{t-\delta}^{t} P(s+\delta) f(x(s)) d s=0 \text { and } \lim _{t \rightarrow \infty} \int_{\beta t}^{t} \frac{Q(s / \beta)}{s} x(s) d s=0
$$

This implies that

$$
\lim _{t \rightarrow \infty}[x(t)+C(t) x(t-\tau)-D(t) x(\alpha t)]=\kappa .
$$

Next, we shall prove that

$$
\lim _{t \rightarrow \infty} x(t) \text { exists and is finite. }
$$

Further, we first show that $|x(t)|$ is bounded. Actually, if $|x(t)|$ is unbounded, then there exists a sequence $\left\{z_{n}\right\}$ such that $z_{n} \rightarrow \infty,\left|x\left(z_{n}^{-}\right)\right| \rightarrow \infty$, as $n \rightarrow \infty$ and

$$
\left|x\left(z_{n}^{-}\right)\right|=\sup _{t_{0} \leq t \leq z_{n}}|x(t)|
$$


where, if $z_{n}$ is not an impulsive point, then $x\left(z_{n}^{-}\right)=x\left(z_{n}\right)$. Thus, we have

$$
\begin{aligned}
& \left|x\left(z_{n}^{-}\right)+C\left(z_{n}^{-}\right) x\left(z_{n}^{-}-\tau\right)-D\left(z_{n}^{-}\right) x\left(\alpha z_{n}^{-}\right)\right| \\
& \quad \geq\left|x\left(z_{n}^{-}\right)\right|-\left|C\left(z_{n}^{-}\right)\right|\left|x\left(z_{n}^{-}-\tau\right)\right|-\left|D\left(z_{n}^{-}\right)\right|\left|x\left(\alpha z_{n}^{-}\right)\right| \\
& \quad \geq\left|x\left(z_{n}^{-}\right)\right|[1-\mu-\varepsilon-\gamma-\lambda] \rightarrow \infty,
\end{aligned}
$$

as $n \rightarrow \infty$, which contradicts (2.20). Therefore, $|x(t)|$ is bounded.

If $\mu=0$ and $\gamma=0$, then $\lim _{t \rightarrow \infty} x(t)=\kappa$, which implies that (2.21) holds. If $0<\mu<1$ and $0<\gamma<1$, then we deduce that $C(t)$ and $D(t)$ are eventually positive or eventually negative. Otherwise, there are two sequences $\left\{w_{k}\right\}$ and $\left\{w_{j}^{*}\right\}$ with $\lim _{k \rightarrow \infty} w_{k}=\infty$ and $\lim _{j \rightarrow \infty} w_{j}^{*}=$ $\infty$ such that $C\left(w_{k}\right)=0$ and $D\left(w_{j}^{*}\right)=0$. Therefore, $C\left(w_{k}\right) \rightarrow 0$ and $D\left(w_{j}^{*}\right) \rightarrow 0$ as $k, j \rightarrow \infty$. It is a contradiction to $\mu>0$ and $\gamma>0$.

Now, we will show that $(2.21)$ holds. By condition $\left(\mathrm{H}_{2}\right)$, we can find a sufficiently large $T_{1}$ such that for $t>T_{1},|C(t)|+|D(t)|<1$. Set

$$
\omega=\liminf _{t \rightarrow \infty} x(t), \quad \theta=\limsup _{t \rightarrow \infty} x(t) .
$$

Then we can choose two sequences $\left\{u_{n}\right\}$ and $\left\{v_{n}\right\}$ such that $u_{n} \rightarrow \infty, v_{n} \rightarrow \infty$ as $n \rightarrow \infty$, and

$$
\lim _{n \rightarrow \infty} x\left(u_{n}\right)=\omega, \quad \lim _{n \rightarrow \infty} x\left(v_{n}\right)=\theta .
$$

For $t>T_{1}$, we consider the following eight possible cases.

Case 1. When $\lim _{t \rightarrow \infty} C(t)=0$ and $-1<D(t)<0$ for $t>T_{1}$, we have

$$
\kappa=\lim _{n \rightarrow \infty}\left[x\left(u_{n}\right)-D\left(u_{n}\right) x\left(\alpha u_{n}\right)\right] \leq \omega+\gamma \theta
$$

and

$$
\kappa=\lim _{n \rightarrow \infty}\left[x\left(v_{n}\right)-D\left(v_{n}\right) x\left(\alpha v_{n}\right)\right] \geq \theta+\gamma \omega .
$$

Thus, we obtain

$$
\omega+\gamma \theta \geq \theta+\gamma \omega
$$

that is,

$$
\omega(1-\gamma) \geq \theta(1-\gamma)
$$

Since $0<\gamma<1$ and $\theta \geq \omega$, it follows that $\theta=\omega$. By (2.20), we obtain

$$
\theta=\omega=\frac{\kappa}{1-\gamma},
$$

which shows that (2.21) holds. 
Case 2. When $\lim _{t \rightarrow \infty} D(t)=0$ and $-1<C(t)<0$ for $t>T_{1}$, we get

$$
\kappa=\lim _{n \rightarrow \infty}\left[x\left(u_{n}\right)+C\left(u_{n}\right) x\left(u_{n}-\tau\right)\right] \leq \omega-\mu \omega
$$

and

$$
\kappa=\lim _{n \rightarrow \infty}\left[x\left(v_{n}\right)+C\left(v_{n}\right) x\left(v_{n}-\tau\right)\right] \geq \theta-\mu \theta,
$$

which leads to

$$
\omega(1-\mu) \geq \theta(1-\mu)
$$

Since $0<\mu<1$ and $\theta \geq \omega$, we conclude that

$$
\theta=\omega=\frac{\kappa}{1-\mu}
$$

which implies that (2.21) holds.

Case 3. $\lim _{t \rightarrow \infty} C(t)=0,0<D(t)<1$ for $t>T_{1}$. The method of proof is similar to the above two cases. Therefore, we omit it.

Case 4 . $\lim _{t \rightarrow \infty} D(t)=0,0<C(t)<1$ for $t>T_{1}$. The method of proof is similar to the above two first cases. Therefore, we omit it.

Case 5. When $-1<D(t)<0$ and $0<C(t)<1$ for $t>T_{1}$, we have

$$
\kappa=\lim _{n \rightarrow \infty}\left[x\left(u_{n}\right)+C\left(u_{n}\right) x\left(u_{n}-\tau\right)-D\left(u_{n}\right) x\left(\alpha u_{n}\right)\right] \leq \omega+\mu \theta+\gamma \theta
$$

and

$$
\kappa=\lim _{n \rightarrow \infty}\left[x\left(v_{n}\right)+C\left(v_{n}\right) x\left(v_{n}-\tau\right)-D\left(v_{n}\right) x\left(\alpha v_{n}\right)\right] \geq \theta+\mu \omega+\gamma \omega
$$

which yields

$$
\omega(1-\mu-\gamma) \geq \theta(1-\mu-\gamma)
$$

Since $0<\mu+\gamma<1$ and $\theta \geq \omega$, we have $\theta=\omega$. Thus

$$
\theta=\omega=\frac{\kappa}{1-\mu-\gamma},
$$

and so (2.21) holds.

Using similar arguments, we can prove that (2.21) also holds for the following cases:

Case 6. $-1<C(t)<0,0<D(t)<1$.

Case 7. $-1<C(t)<0,-1<D(t)<0$.

Case 8. $0<C(t)<1,0<D(t)<1$.

Summarizing the above investigation, we conclude that (2.21) holds and so the proof is completed.

Theorem 2.2 Let conditions $\left(\mathrm{H}_{1}\right)-\left(\mathrm{H}_{4}\right)$ of Theorem 2.1 hold. Then every oscillatory solution of (1.1) tends to zero as $t \rightarrow \infty$. 
Corollary 2.1 Assume that $\left(\mathrm{H}_{3}\right)$ holds and

$$
\limsup _{t \rightarrow \infty}\left[\int_{t-\delta}^{t+\delta} P(s+\delta) d s+\int_{\beta t}^{t+\delta} \frac{Q(s / \beta)}{s} d s\right]<2
$$

and

$$
\limsup _{t \rightarrow \infty}\left[\int_{t-\delta}^{t / \beta} P(s+\delta) d s+\int_{\beta t}^{t / \beta} \frac{Q(s / \beta)}{s} d s\right]<2
$$

Then every solution of the equation

$$
\left\{\begin{aligned}
x^{\prime}(t)+ & P(t) x(t-\delta)+\frac{Q(t)}{t} x(\beta t)=0, \quad 0<t_{0} \leq t, t \neq t_{k} \\
x\left(t_{k}\right)= & b_{k} x\left(t_{k}^{-}\right)+\left(1-b_{k}\right)\left(\int_{t_{k}-\delta}^{t_{k}} P(s+\delta) x(s) d s\right. \\
& \left.+\int_{\beta t_{k}}^{t_{k}} \frac{Q(s / \beta)}{s} x(s) d s\right), \quad k=1,2,3, \ldots
\end{aligned}\right.
$$

tends to a constant as $t \rightarrow \infty$.

Corollary 2.2 The conditions (2.23) and (2.24) imply that every solution of the equation

$$
x^{\prime}(t)+P(t) x(t-\delta)+\frac{Q(t)}{t} x(\beta t)=0, \quad 0<t_{0} \leq t,
$$

tends to a constant as $t \rightarrow \infty$.

Theorem 2.3 The conditions $\left(\mathrm{H}_{1}\right)-\left(\mathrm{H}_{4}\right)$ of Theorem 2.1 together with

$$
\int_{t_{0}}^{\infty} P(s+\delta) d s=\infty, \quad \int_{t_{0}}^{\infty} \frac{Q(s / \beta)}{s} d s=\infty
$$

imply that every solution of (1.1) tends to zero as $t \rightarrow \infty$.

Proof From Theorem 2.2, we only have to prove that every nonoscillatory solution of (1.1) tends to zero as $t \rightarrow \infty$. Without loss of generality, we assume that $x(t)$ is an eventually positive solution of (1.1). As in the proof of Theorem 2.1, (1.1) can be written as in the form (2.18). Integrating from $t_{0}$ to $t$ both sides of the first equation of (2.18), one has

$$
\int_{t_{0}}^{t} P(s+\delta) f(x(s)) d s+\int_{t_{0}}^{t} \frac{Q(s / \beta)}{s} x(s) d s=y\left(t_{0}\right)-y(t)-\sum_{t_{0}<t_{k}<t}\left(1-b_{k}\right) y\left(t_{k}^{-}\right) .
$$

Applying (2.19) and $\left(\mathrm{H}_{3}\right)$, we have

$$
\int_{t_{0}}^{\infty} P(s+\delta) f(x(s)) d s<\infty \text { and } \int_{t_{0}}^{\infty} \frac{Q(s / \beta)}{s} x(s) d s<\infty .
$$

This, together with (2.27), implies that $\liminf _{t \rightarrow \infty} f(x(t))=0$ and $\liminf _{t \rightarrow \infty} x(t)=0$. By Theorem 2.1, $\lim _{t \rightarrow \infty} x(t)=0$. This completes the proof. 
Corollary 2.3 Assume that (2.1), (2.2), (2.4), (2.5), and (2.27) hold. Then every solution of the equation

$$
[x(t)+C(t) x(t-\tau)-D(t) x(\alpha t)]^{\prime}+P(t) f(x(t-\delta))+\frac{Q(t)}{t} x(\beta t)=0,
$$

$0<t_{0} \leq t$, tends to zero as $t \rightarrow \infty$.

\section{Examples}

In this section, we present two examples to illustrate our results.

Example 3.1 Consider the following mixed type neutral differential equation with impulsive perturbations:

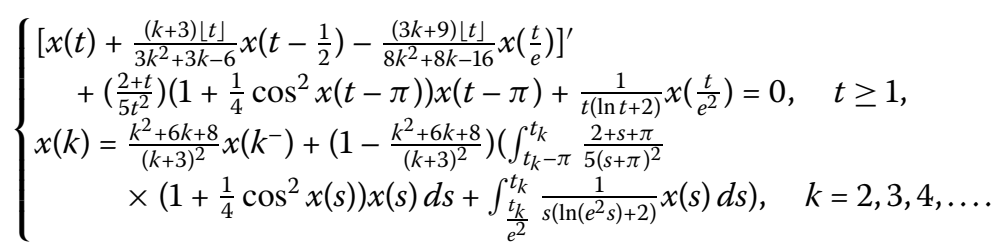

Here $C(t)=((k+3)\lfloor t\rfloor) /\left(3 k^{2}+3 k-6\right), D(t)=((3 k+9)\lfloor t\rfloor) /\left(8 k^{2}+8 k-16\right), P(t)=(2+$ $t) /\left(5 t^{2}\right), Q(t)=1 /(\ln t+2), t \in[k-1, k), b_{k}=\left(k^{2}+6 k+8\right) /\left((k+3)^{2}\right), t_{0}=1, k=2,3,4, \ldots$, $f(x)=x\left(1+\left((1 / 4)\left(\cos ^{2} x\right)\right)\right), \tau=1 / 2, \delta=\pi, \alpha=1 / e$, and $\beta=1 / e^{2}$. We can find that

(i) $|x| \leq\left|\left(1+\frac{1}{4} \cos ^{2} x\right) x\right| \leq \frac{5}{4}|x|, x \in \mathbb{R},\left(1+\frac{1}{4} \cos ^{2} x\right) x^{2}>0$ for $x \neq 0$;

(ii) $\lim _{t \rightarrow \infty}|C(t)|=\frac{1}{3}=\mu<1, \lim _{t \rightarrow \infty}|D(t)|=\frac{3}{8}=\gamma<1$ with $\mu+\gamma=\frac{17}{24}<1$, and $C(k)=\frac{k^{2}+6 k+8}{(k+3)^{2}} C\left(k^{-}\right), D(k)=\frac{k^{2}+6 k+8}{(k+3)^{2}} D\left(k^{-}\right) ;$

(iii) $t_{k}-(1 / 2)$ and $(1 / e) t_{k}$ are not impulsive points, $0<\left(k^{2}+6 k+8\right) /\left((k+3)^{2}\right) \leq 1$ for $k=1,2, \ldots$, and

$$
\sum_{k=1}^{\infty}\left(1-\frac{k^{2}+6 k+8}{(k+3)^{2}}\right)=\sum_{k=1}^{\infty} \frac{1}{(k+3)^{2}}<\infty ;
$$

(iv)

$$
\left\{\begin{array}{c}
\lim \sup _{t \rightarrow \infty}\left[\int_{t-\delta}^{t+\delta} P(s+\delta) d s+\int_{\beta t}^{t+\delta} \frac{Q(s / \beta)}{s} d s\right. \\
\left.\quad+\mu\left(1+\frac{P(t+\tau+\delta)}{P(t+\delta)}\right)+\gamma\left(1+\frac{P((t / \alpha)+\delta)}{\alpha P(t+\delta)}\right)\right]=\frac{17}{12}<\frac{8}{5}
\end{array}\right.
$$

and

$$
\left\{\begin{array}{l}
\lim \sup _{t \rightarrow \infty}\left[\int_{t-\delta}^{t / \beta} P(s+\delta) d s+\int_{\beta t}^{t / \beta} \frac{Q(s / \beta)}{s} d s\right. \\
\left.\quad+\mu\left(1+\frac{t Q(t+\tau) / \beta)}{(t+\tau) Q(t / \beta)}\right)+\gamma\left(1+\frac{Q(t /(\alpha \beta))}{Q(t / \beta)}\right)\right]=\frac{109}{60}<2 .
\end{array}\right.
$$

Hence, by (i)-(iv) all assumptions of Theorem 2.1 are satisfied. Therefore, we conclude that every solution of (3.1) tends to a constant as $t \rightarrow \infty$. 
Example 3.2 Consider the following mixed type neutral differential equation with impulsive perturbations

$$
\left\{\begin{aligned}
& {[x(t)+}\left.\frac{(12 k+16)\lfloor t\rfloor}{54 k^{2}+27 k-27} x\left(t-\frac{2}{3}\right)-\frac{(12 k+16)\lfloor t\rfloor}{42 k^{2}+21 k-21} x\left(\frac{t}{2 e^{3}}\right)\right]^{\prime} \\
& \quad+\left(\frac{2 t+1}{(4 t+3)^{2}}\right)\left(1+\frac{2}{5} \sin ^{2} x\left(t-\frac{\pi}{2}\right)\right) x\left(t-\frac{\pi}{2}\right)+\frac{4}{t(2 \ln t+3)} x\left(\frac{t}{3 e}\right)=0, \quad t \geq 1, \\
& x(k)= \frac{6 k^{2}+17 k+7}{6 k^{2}+17 k+12} x\left(k^{-}\right)+\left(1-\frac{6 k^{2}+17 k+7}{6 k^{2}+17 k+12}\right)\left(\int_{t_{k}-\frac{\pi}{2}}^{t} \frac{2 s+1+\pi}{(4 s+3+2 \pi)^{2}}\right. \\
& \quad\left.\times\left(1+\frac{2}{5} \sin ^{2} x(s)\right) x(s) d s+\int_{\frac{t_{k}}{3 e}}^{t_{k}} \frac{4}{s(2 \ln (3 e s)+3)} x(s) d s\right), \quad k=2,3,4, \ldots
\end{aligned}\right.
$$

Here $C(t)=((12 k+16)\lfloor t\rfloor) /\left(54 k^{2}+27 k-27\right), D(t)=((12 k+16)\lfloor t\rfloor) /\left(42 k^{2}+21 k-21\right)$, $P(t)=(2 t+1) /\left((4 t+3)^{2}\right), Q(t)=4 /(2 \ln t+3), t \in[k-1, k), b_{k}=\left(6 k^{2}+17 k+7\right) /\left(6 k^{2}+\right.$ $17 k+12), t_{0}=1, k=2,3,4, \ldots, f(x)=x\left(1+\left((2 / 5) \sin ^{2} x\right)\right), \tau=2 / 3, \delta=\pi / 2, \alpha=1 /\left(2 e^{3}\right)$, and $\beta=1 /(3 e)$. We can show that

(i) $|x| \leq\left|\left(1+\frac{2}{5} \sin ^{2} x\right) x\right| \leq \frac{7}{5}|x|, x \in \mathbb{R},\left(1+\frac{2}{5} \sin ^{2} x\right) x^{2}>0$ for $x \neq 0$;

(ii) $\lim _{t \rightarrow \infty}|C(t)|=\frac{2}{9}=\mu<1, \lim _{t \rightarrow \infty}|D(t)|=\frac{2}{7}=\gamma<1$ with $\mu+\gamma=\frac{32}{63}<1$, and $C(k)=\frac{6 k^{2}+17 k+7}{6 k^{2}+17 k+12} C\left(k^{-}\right), D(k)=\frac{6 k^{2}+17 k+7}{6 k^{2}+17 k+12} D\left(k^{-}\right) ;$

(iii) $t_{k}-(2 / 3)$ and $\left(1 /\left(2 e^{3}\right)\right) t_{k}$ are not impulsive points, $0<\left(6 k^{2}+17 k+7\right) /\left(6 k^{2}+17 k+12\right) \leq 1$ for $k=1,2, \ldots$, and

$$
\sum_{k=1}^{\infty}\left(1-\frac{6 k^{2}+17 k+7}{6 k^{2}+17 k+12}\right)=\sum_{k=1}^{\infty} \frac{5}{6 k^{2}+17 k+12}<\infty
$$

(iv)

$$
\left\{\begin{array}{l}
\lim \sup _{t \rightarrow \infty}\left[\int_{t-\delta}^{t+\delta} P(s+\delta) d s+\int_{\beta t}^{t+\delta} \frac{Q(s / \beta)}{s} d s\right. \\
\left.\quad+\mu\left(1+\frac{P(t+\tau+\delta)}{P(t+\delta)}\right)+\gamma\left(1+\frac{P((t / \alpha)+\delta)}{\alpha P(t+\delta)}\right)\right]=\frac{64}{63}<\frac{10}{7}
\end{array}\right.
$$

and

$$
\left\{\begin{array}{l}
\lim \sup _{t \rightarrow \infty}\left[\int_{t-\delta}^{t / \beta} P(s+\delta) d s+\int_{\beta t}^{t / \beta} \frac{Q(s / \beta)}{s} d s\right. \\
\left.\quad+\mu\left(1+\frac{t Q((t+\tau)(\beta)}{(t+\tau) Q(t / \beta)}\right)+\gamma\left(1+\frac{Q(t /(\alpha \beta))}{Q(t / \beta)}\right)\right]=1.2781996<2
\end{array}\right.
$$

(v)

$$
\int_{1}^{\infty} P(s+\delta) d s=\int_{1}^{\infty} \frac{2 s+1+\pi}{(4 s+3+2 \pi)^{2}} d s=\infty
$$

and

$$
\int_{1}^{\infty} \frac{Q(s / \beta)}{s} d s=\int_{1}^{\infty} \frac{4}{s(2 \ln (3 e s)+3)} d s=\infty .
$$

Hence, all assumptions of Theorem 2.3 are satisfied and therefore every solution of (3.2) tends to zero as $t \rightarrow \infty$. 
Authors' contributions

All authors contributed equally in this article. They read and approved the final manuscript.

\section{Author details}

'Nonlinear Dynamic Analysis Research Center, Department of Mathematics, Faculty of Applied Science, King Mongkut's University of Technology North Bangkok, Bangkok, 10800, Thailand. ${ }^{2}$ Department of Mathematics, University of loannina, Ioannina, 451 10, Greece. ${ }^{3}$ Nonlinear Analysis and Applied Mathematics (NAAM)-Research Group, Department of Mathematics, Faculty of Science, King Abdulaziz University, P.O. Box 80203, Jeddah, 21589, Saudi Arabia.

\section{Acknowledgements}

We would like to thank the reviewers for their valuable comments and suggestions on the manuscript. This research was funded by King Mongkut's University of Technology North Bangkok. Contract no. KMUTNB-GOV-57-08.

Received: 29 August 2014 Accepted: 9 December 2014 Published: 22 Dec 2014

\section{References}

1. Bainov, DD, Simeonov, PS: Systems with Impulse Effect. Ellis Horwood, Chichester (1989)

2. Lakshmikantham, V, Bainov, DD, Simeonov, PS: Theory of Impulsive Differential Equations. World Scientific, Singapore (1989)

3. Samoilenko, AM, Perestyuk, NA: Impulsive Differential Equations. World Scientific, Singapore (1995)

4. Benchohra, M, Henderson, J, Ntouyas, SK: Impulsive Differential Equations and Inclusions, vol. 2. Hindawi Publishing Corporation, New York (2006)

5. Bainov, DD, Dinitrova, MB, Dishliev, AB: Oscillation of the solutions of impulsive differential equations and inequalities with a retarded argument. Rocky Mt. J. Math. 28, 25-40 (1998)

6. Luo, Z, Shen, J: Stability and boundedness for impulsive differential equations with infinite delays. Nonlinear Anal. 46 , 475-493 (2001)

7. Liu, X, Shen, J: Asymptotic behavior of solutions of impulsive neutral differential equations. Appl. Math. Lett. 12, 51-58 (1999)

8. Shen, J, Liu, Y, Li, J: Asymptotic behavior of solutions of nonlinear neutral differential equations with impulses. J. Math. Anal. Appl. 332, 179-189 (2007)

9. Shen, J, Liu, Y: Asymptotic behavior of solutions for nonlinear delay differential equation with impulses. J. Appl. Math. Comput. 213, 449-454 (2009)

10. Wei, G, Shen, J: Asymptotic behavior of solutions of nonlinear impulsive delay differential equations with positive and negative coefficients. Math. Comput. Model. 44, 1089-1096 (2006)

11. Luo, J, Debnath, L: Asymptotic behavior of solutions of forced nonlinear neutral delay differential equations with impulses. J. Appl. Math. Comput. 12, 39-47 (2003)

12. Jiang, F, Sun, J: Asymptotic behavior of neutral delay differential equation of Euler form with constant impulsive jumps. Appl. Math. Comput. 219, 9906-9913 (2013)

13. Pandian, S, Balachandran, Y: Asymptotic behavior results for nonlinear impulsive neutral differential equations with positive and negative coefficients. Bonfring Int. J. Data Min. 2, 13-21 (2012)

14. Wang, QR: Oscillation criteria for first-order neutral differential equations. Appl. Math. Lett. 8, 1025-1033 (2002)

15. Tariboon, J, Thiramanus, P: Oscillation of a class of second-order linear impulsive differential equations. Adv. Differ. Equ. 2012, 205 (2012)

16. Jiang, F, Shen, J: Asymptotic behavior of solutions for a nonlinear differential equation with constant impulsive jumps. Acta Math. Hung. 138, 1-14 (2013)

17. Jiang, F, Shen, J: Asymptotic behaviors of nonlinear neutral impulsive delay differential equations with forced term. Kodai Math. J. 35, 126-137 (2012)

18. Gunasekar, T, Samuel, FP, Arjunan, MM: Existence results for impulsive neutral functional integrodifferential equation with infinite delay. J. Nonlinear Sci. Appl. 6, 234-243 (2013)

19. Kumar, P, Pandey, DN, Bahuguna, D: On a new class of abstract impulsive functional differential equations of fractional order. J. Nonlinear Sci. Appl. 7, 102-114 (2014)

20. Samuel, FP, Balachandran, K: Existence of solutions for quasi-linear impulsive functional integrodifferential equations in Banach spaces. J. Nonlinear Sci. Appl. 7, 115-125 (2014)

21. Guan, $\mathrm{K}$, Shen, J: Asymptotic behavior of solutions of a first-order impulsive neutral differential equation in Euler form. Appl. Math. Lett. 24, 1218-1224 (2011)

10.1186/1687-1847-2014-327

Cite this article as: Tariboon et al.: Asymptotic behavior of solutions of mixed type impulsive neutral differential equations. Advances in Difference Equations 2014, 2014:327 